\title{
A Micro-CT Study of Different Regions of the Atlas
}

\author{
Un Estudio de Micro-CT de Diferentes Regiones del Atlas
}

\begin{abstract}
Zhenhua Cao ${ }^{1,2}$; Baoke Su${ }^{3}$;Wei Wang ${ }^{4}$; Zhijun $\mathrm{Li}^{3}$; Haiyan Wang ${ }^{3}$; Xing Wang ${ }^{3}$; Shaojie Zhang ${ }^{3}$; Yidan Wang ${ }^{3}$; Huanhuan Guan'; Mingjie Gao ${ }^{3}$; Zhiqiang Wang ${ }^{3}$; Yunfeng Zhang²; Xiaohe Li $^{3}$ \& Yan Wang
\end{abstract}

CAO, Z.; SU, B.; WANG, W.; LI, Z.; WANG, H.; WANG, X.; ZHANG, S.; WANG, Y.; GUAN, H.; GAO, M.; WANG, Z.; ZHANG, Y.; LI, X. \& WANG, Y. A micro-CT study of different regions of the atlas. Int. J. Morphol., 38(2):477-480, 2020.

SUMMARY: This study aimed to examine the differences in bone microarchitecture between different regions of the atlas in 28 dry atlas specimens using micro-CT, in order to explain the mechanism of the predilection sites of atlas fractures from the morphological point of view. A total of 28 dry specimens of intact adult atlas were randomly selected, scanned by micro-CT, and divided into a region from the anterior arch midpoint (AAM) to the lateral masses (LM), a LM region, and a region from the LM to the posterior arch midpoint (PAM). Trabecular thickness, separation, number, connectivity, and structure model index were measured for each of the three regions using the built-in software of the CT scanner. Trabecular thickness was all measured to be $0.11 \pm 0.00 \mathrm{~mm}$ for AAM to LM, LM, and LM to PAM. Trabecular separation: AAM to LM > LM to PAM > LM. Trabecular number: LM > LM to PAM > AAM to LM. Connectivity: $\mathrm{LM}>\mathrm{LM}$ to $\mathrm{PAM}>\mathrm{AAM}$ to LM. Structure model index: $\mathrm{LM}>\mathrm{LM}$ to PAM > AAM to LM. A lower trabecular number and connectivity and higher trabecular separation were seen in the anterior and posterior arches of the atlas, in which higher fracture rates were reported. By contrast, a higher trabecular number and connectivity and lower trabecular separation were seen in the lateral masses, in which lower fracture rates were reported.

KEY WORDS: Micro-CT; Atlas; Fracture; Trabeculae.

\section{INTRODUCTION}

The atlas is located at the junction of the skull and spine, serving as a connecting link in the occipital-atlantoaxial complex. It is adjacent to the "vital knot", medulla oblongata. The vertebral artery enters the cranium through the transverse foramen of the atlas. Therefore, atlas fractures are life-threatening injuries. Adult atlas fractures account for $25 \%$ of upper cervical spine injuries, $2 \%$ to $13 \%$ of cervical spine fractures, and $1 \%$ to $2 \%$ of spinal fractures (Hadley et al., 1988; Kakarla et $a l ., 2010)$. Many researchers have investigated the classification and treatment of atlas fractures, whereas rare studies have examined the predilection sites of atlas fractures from the morphological point of view. Therefore, this study aimed to analyze the bone microarchitecture of different regions of the atlas by using micro-CT, in order to explain the mechanism of the predilection sites of atlas fractures.

\section{MATERIAL AND METHOD}

Specimens and tools. A total of 28 dry specimens of intact atlas, whose gender and age were unknown, provided by the Department of Human Anatomy, Inner Mongolia Medical University were randomly selected. Specimens with defects and deformities were excluded. Hiscan XM Micro CT (Hounsfield, Suzhou, China) and its analyzer software (Hiscan Analyzer) were used with the following parameters: slice thickness $0.05 \mathrm{~mm}$, slice gap $0.05 \mathrm{~mm}$, single exposure time $50 \mathrm{~ms}$, tube voltage $60 \mathrm{KV}$, current $134 \mathrm{uA}$, matrix $2000 \times 1600$, field of view $10 \times 8 \mathrm{~cm}$, and pixel size $0.05 \times 0.05$. The acquired DICOM images were processed and stored by the Lenovo P320 workstation provided by Haysfield (Suzhou, China).

Methods: The 28 atlas specimens were numbered, scanned with Hiscan XM Micro CT, and divided into a region from the anterior arch midpoint (AAM) to the la-

\footnotetext{
${ }^{1}$ Postdoctoral Workstation, Department of Orthopaedics, Chinese People's Liberation Army General Hospital,28 Fuxing Road, 100853 Beijing, People's Republic of China.

${ }^{2}$ The second Affiliated Hospital of Inner Mongolia Medical University, Hohhot, 010050, China.

${ }^{3}$ The department of anatomy (Digital Medical Center), Inner Mongolia Medical University, Hohhot, 010059, China.

${ }^{4}$ The department Emergency, Inner Mongolia People's Hospital, Hohhot, 010017, China.

${ }^{5}$ Department of Orthopaedics, Chinese People's Liberation Army General Hospital,28 Fuxing Road, 100853 Beijing, People's Republic of China. Cao, Z. and Su, B. are co-first authors.
} 
teral masses (LM), a LM region, and a region from the LM to the posterior arch midpoint (PAM) (Fig 1). The following parameters were measured for each of the three regions using the built-in Hiscan Analyzer software: total volume (TV), bone volume (BV), trabecular thickness (Tb.Th), trabecular separation (Tb.Sp), trabecular number (Tb.N), trabecular connectivity (Conn), and trabecular structure model index (SMI). The definition and significance of each parameter are shown in Table I. The reconstructed trabeculae of the atlas are shown in Figure 2. Each parameter was measured 3 times and the average was recorded.
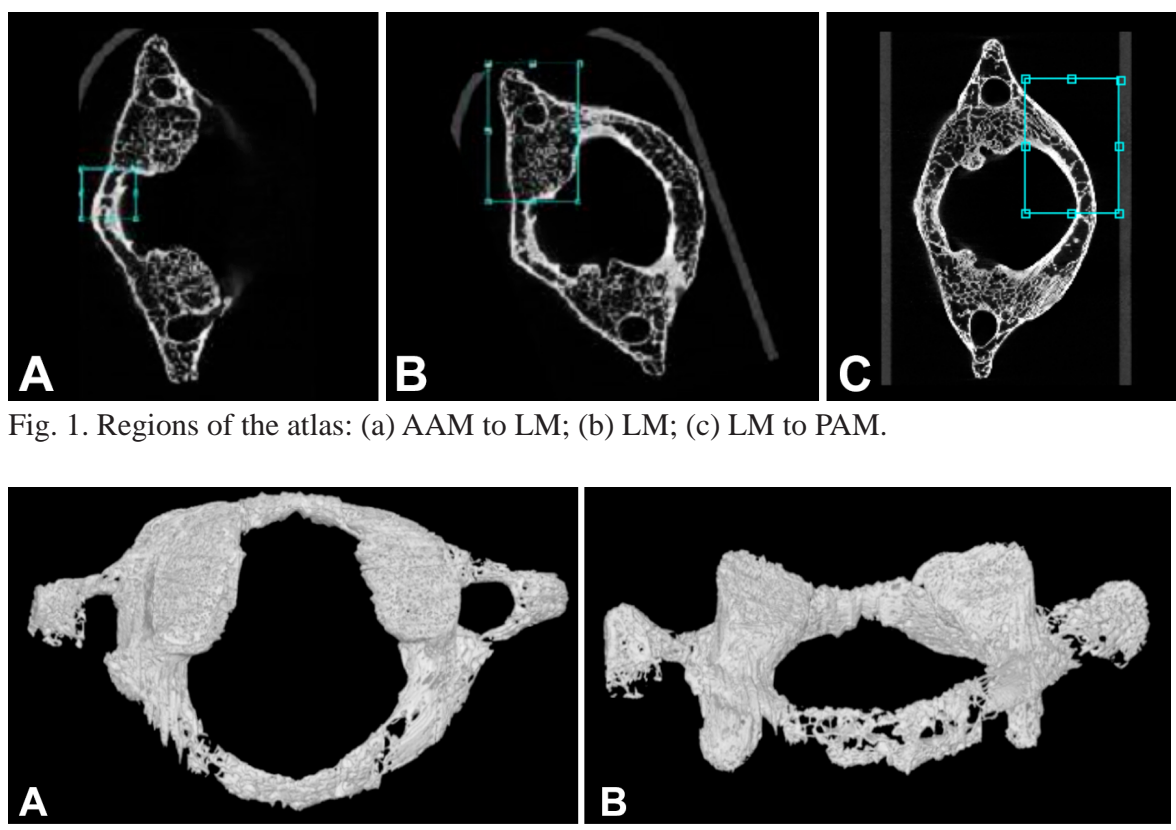

Fig. 2. Reconstructed trabeculae of the atlas.

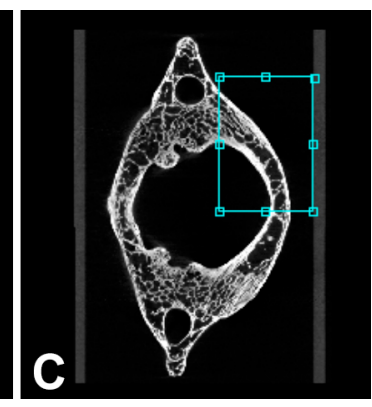

Statistical analysis. Data were analyzed using SPSS 13.0. Normally distributed measurement data were expressed as mean \pm standard deviation $( \pm s)$ and analyzed by analysis of variance. An a $=0.05(\mathrm{P}$ $<0.05)$ was considered significant.

\section{RESULTS}

As shown in Table II, trabecular thickness was all measured to be $0.11 \pm 0.00 \mathrm{~mm}$ for AAM to LM, LM, and LM to PAM. Trabecular separation: AAM to LM $>$ LM to PAM > LM. Trabecular number: $\mathrm{LM}>\mathrm{LM}$ to $\mathrm{PAM}>\mathrm{AAM}$ to LM. Connectivity: LM > LM to PAM > AAM to LM. Structure model index: LM > LM to PAM > AAM to LM.

Table I. Morpometric parameters

\begin{tabular}{|c|c|}
\hline arameter & Definition and significance \\
\hline otal volume (TV) & Volume of the region of inter est $\left(\mathrm{mm}^{3}\right)$ \\
\hline one volume (BV) & Trabecular volume in the region of interest $\left(\mathrm{mm}^{3}\right)$ \\
\hline abecular thickness (Tb.Th) & Average thickness of the trabeculae (_m), which decreases in osteoporosis \\
\hline abecular number (Tb.N) & $\begin{array}{l}\text { Number of intersections between bone and non-bone tissues within a given length (n } \\
{ }^{1} \text { ), which decreases in osteoporosis }\end{array}$ \\
\hline abecular separation (Tb.Sp) & Average distance between trabeculae (_m), which increases in osteoporosis \\
\hline abecular c onnectivity (Conn) & $\begin{array}{l}\text { Degree of interconnection between trabeculae }\left(\mathrm{mm}^{-3}\right) \text {, the decrease of which may res } \\
\text { in bone disease }\end{array}$ \\
\hline abecular structure model index (SMI) & Degree of rod_like versus plate_like trabeculae, which increases in osteoporosis \\
\hline
\end{tabular}

Table II. Bone microarchitecture measurements in different regions of the atlas $(n=28)$

\begin{tabular}{lccccc}
\hline & AAM to LM & LM & LM to PAM & F & P \\
\hline V & $20.50 \pm 4.59$ & $235.39 \pm 43.92$ & $46.18 \pm 10.79$ & & \\
iV & $18.52 \pm 4.95$ & $225.86 \pm 52.36$ & $42.46 \pm 12.91$ & & \\
'b_Th & $0.11 \pm 0.00$ & $0.11 \pm 0.00$ & $0.11 \pm 0.00$ & 1.12 & 0.35 \\
'b_N & $2.44 \pm 0.44$ & $2.95 \pm 0.25$ & $2.87 \pm 0.51$ & 9.50 & 0.00 \\
'b_Sp & $0.32 \pm 0.76$ & $0.23 \pm 0.29$ & $0.25 \pm 0.51$ & 9.98 & 0.00 \\
'onn & $179.31 \pm 91.27$ & $4981.15 \pm 1640.44$ & $518.13 \pm 173.41$ & 126.34 & 0.00 \\
MI & $2.53 \pm 0.32$ & $2.81 \pm 0.15$ & $152.73 \pm 0.37$ & 2.61 & 0.06 \\
\hline
\end{tabular}

Note: The units of each parameter are shown in Table I. $\mathrm{P}<0.05$ was considered statistically significant. 


\section{DISCUSSION}

Strengths of micro-CT in evaluating atlas microarchitecture. The study of trabecular architecture currently is based on imaging. Traditionally, the imaging of trabeculae is performed by sectioning (Wan et al., 2007), which, however, is associated with large bone damage, difficult analysis, long time requirement, and low accuracy. With the rapid development of computer and imaging technology, micro-CT is able to offer high resolution (up to micron level), image quality, and accuracy with a short time requirement for the structural analysis of trabeculae. Clear, separable trabecular images obtained by micro-CT can be used for two-dimensional image analysis or three-dimensional model analysis to facilitate the observation of trabecular changes and analyze the state of bone tissue (Cui et al., 2012). For example, microCT has been used to analyze the anatomy of the trabecular bone of the jaw by Jin et al. (2011) to examine the bone mineral density and trabecular number, thickness, and morphology around the dental implant in Beagle dogs by Zhang et al. (2017) and to determine the effect of a Chinese medicine, Qiangguyin, on the trabecular number, thickness, and morphology of bone callus in ovariectomized rats by Yao et al. (2016).

Predilection sites of atlas fractures. The atlas consists of an anterior arch, a posterior arch, and two lateral masses. According to Landells and Van Peteghem's classification (Landells \& Van Peteghem, 1988). Type I fracture involves the anterior or posterior arch alone. Type II fracture involves fractures of both the anterior and posterior arches. Type III fracture is a fracture of the lateral mass with or without a fracture of the arch. Of the 33 cases of fresh unstable atlas fractures collected by Li et al. (2008) for surgical strategy and efficacy analysis, $3(9 \%)$ were Type I, 21 (64 \%) were Type II, and $9(27 \%)$ were Type II; and anterior and/or posterior arch fractures accounted for $73 \%$. Of the 35 cases of atlas fractures reviewed by Landells \& Van Peteghem. Sixteen (46 $\%)$ were Type I, 13 (37\%) were Type II, and $6(17 \%)$ were Type III; and anterior and/or posterior arch fractures accounted for $83 \%$. It can be seen that atlas fractures most frequently involve the anterior and/or posterior arch, followed by the lateral masses.

Differences in bone microarchitecture between different regions of the atlas. The trabeculae of the atlas are interconnected to form an irregular reticular arrangement. Trabecular thickness was all measured to be $0.11 \pm 0.00 \mathrm{~mm}$ for AAM to LM, LM, and LM to PAM. Trabecular separation: AAM to LM > LM to PAM > LM. Trabecular number: $\mathrm{LM}>$ LM to PAM > AAM to LM. Connectivity: $\mathrm{LM}>\mathrm{LM}$ to PAM > AAM to LM. Structure model index: LM > LM to PAM > AAM to LM.
Clinical significance of microarchitecture measurements in atlas fractures. Changes in trabecular architecture are also an important factor in osteoporosis, which, in turn, can easily lead to fracture. Greater trabecular thickness, number, and connectivity and smaller trabecular separation contribute to lower fracture rates.

Outlook and limitations. In this study, the microarchitecture of the atlas was analyzed using micro-CT with high resolution, image quality, and accuracy. However, as the gender and age of the specimens were unknown, the differences in the degree of osteoporosis due to gender and age cannot be excluded. Moreover, this study did not explore the differences in atlas fracture sites caused by external forces in different directions, but simply compared the bone microarchitecture of different regions of the atlas to identify the predilection sites of atlas fractures based on trabecular number, thickness, separation, and connectivity. In future research, it is therefore important to consider gender, age, and external forces in different directions when examining the predilection sites of atlas fractures.

\section{CONCLUSION}

In this study, bone microarchitecture of different regions of the atlas was examined in 28 dry atlas specimens using micro-CT. A lower trabecular number and connectivity and higher trabecular separation were seen in the anterior and posterior arches, in which higher fracture rates were reported. By contrast, a higher trabecular number and connectivity and lower trabecular separation were seen in the lateral masses, in which lower fracture rates were reported. In summary, atlas fractures most frequently involve the anterior arch, followed by the posterior arch, and least frequently involve the lateral masses. This is consistent with clinical observations. This study explains the predilection sites of atlas fractures from the morphological point of view.

FUNDING. National natural science foundation of China (81460330;8156034;81260269);Inner Mongolia department of education youth science and technology talents project (njyt-15-b05);Science and technology program of Inner Mongolia autonomous region (2016); Science and technology innovation guidance project of Inner Mongolia autonomous region (2017); Natural science foundation of the Inner Mongolia autonomous region (2016ms08131); Fund for returned students of Inner Mongolia department of human 
resources and social security;Inner Mongolia medical university undergraduate talent cultivation project (2018/ 2019);Inner Mongolia grassland yingcai team;Science and technology program of Inner Mongolia autonomous region (2019)

CAO, Z.; SU, B.; WANG, W.; LI, Z.; WANG, H.; WANG, X.; ZHANG, S.; WANG, Y.; GUAN, H.; GAO, M.; WANG, Z.; ZHANG, Y.; LI, X. \& WANG, Y. Un estudio de micro-CT de diferentes regiones del atlas. Int. J. Morphol., 38 (2):477-480, 2020.

RESUMEN: Este estudio tuvo como objetivo examinar las diferencias en la microarquitectura ósea entre diferentes regiones del atlas en 28 muestras de atlas secas utilizando micro-CT, con el fin de informar el área de mayor frecuencia en las fracturas de atlas, desde el punto de vista morfológico. Se seleccionaron al azar un total de 28 muestras secas de atlas adultos intactas las que se escanearon por micro-CT y se dividieron en una región desde el punto medio del arco anterior (MAA) hasta las masas laterales (ML), una región ML y una región desde el ML hasta el punto medio del arco posterior (MAP). Se midió el grosor trabecular, la separación, el número, la conectividad y el índice del modelo de estructura para cada una de las tres regiones utilizando el software incorporado del escáner CT. El grosor trabecular se midió en 0,11 $\pm 0,00 \mathrm{~mm}$ para MAA a ML, ML y ML a MAP. Separación trabecular: MAA a ML> ML a MAP> ML. Número trabecular: ML > ML a MAP> MAA a ML. Conectividad: ML> ML a MAP> MAA a ML. Índice del modelo de estructura: ML> ML a MAP> MAA a ML. Se observó un menor número de estructuras trabeculares y conectividad y una mayor separación trabecular en los arcos anterior y posterior del atlas, en los que se informaron tasas de fracturas más altas. Por el contrario, se observó un mayor número de estructuras trabeculares y conectividad, y una menor separación trabecular en las masas laterales, en las que se observó un número menor de fracturas.

PALABRAS CLAVE: Micro-CT; Atlas; Fractura; Trabéculas.

\section{REFERENCES}

Cui, Z.; Xu, Y.; Tang, H.; Fan, X. \& Guo, Y. The research progress of microCT in osteoporosis. Chin. J. Osteoporos., 18(1):87-9, 2012.

Hadley, M. N.; Dickman, C. A.; Browner, C. M. \& Sonntag, V. K. Acute traumatic atlas fractures: management and long term outcome. Neurosurgery, 23(1):31-5, 1988.

Jin, G. C.;Zhang, X. Y.; Jiang, L.; Li, X. L.; Zhang, D.; Li, W. X.; Kim, H. J. \& Jin, D. C. Measurement of anatomical structure of jaw bone trabecula with micro-CT and its accuracy evaluation. J. Jilin Univ. Med. Ed., 37(5):833-7, 2011

Kakarla, U. K.; Chang, S. W.; Theodore, N. \& Sonntag, V. K. H. Atlas fractures. Neurosurgery, 66(3 Suppl.):60-7, 2010.

Landells, C. D. \& Van Peteghem, P. K. Fractures of the atlas: classification, treatment and morbidity. J. Spine (Phila Pa 1976), 13(5):450-2, 1988.

Li, K. K.; Yao, M. \& Zhang, Y. D. Surgical strategy and efficacy analysis of fresh unstable atlas fracture. Chin. J. Bone Joint Inj., 33(05):449-53, 2008.
Wan, S. Y.; Lei, W.; Wu, Z. X.; Lü., R.; Wang, J.; Li, B.; Fu, S. C. \& Zhan, C. Micro-CT Evaluation and histological analysis of screw-bone interface of expansile pedicle screw in osteoporotic sheep. Zhonghua Wai Ke Za Zhi, 45(18):1271-3, 2007.

Yao, J. L.; Wang, B.; Wu, P.; Liu, K. \& Shi, X. L. Study on micro-ct changes of bone trabecular bone healing in ovariectomized rats. J. Chin. Osteoporos., 22(11):1395-8, 2016.

Zhang, H. G.; Sun, W. G.; Liu, X. H.; Jiang, Y.; Zhou, W. M. \& Gao, W. J. Preliminary study on micro-CT analysis of bone trabeculae around implants and corresponding ROI selection. J. Anhui Med. Univ., 52(11):1721-5, 2017.

Corresponding author:

Yan Wang

Department of Orthopaedics,

Chinese People's Liberation Army General Hospital

28 Fuxing Road

100853 Beijing

CHINA

E-mail: yanwang301@163.com

Corresponding author:

Xiaohe Li

Department of Anatomy

(Digital Medical Center)

Inner Mongolia Medical University

Hohhot 010059

CHINA

E-mail:798242742@qq.com

Received: 24-07-2019

Accepted: 09-09-2019 